\title{
ASYMPTOTIC BEHAVIOR OF A COMPETITIVE SYSTEM OF LINEAR FRACTIONAL DIFFERENCE EQUATIONS
}

\author{
M. R. S. KULENOVIĆ AND M. NURKANOVIĆ \\ Received 18 July 2005; Revised 3 April 2006; Accepted 5 April 2006
}

We investigate the global asymptotic behavior of solutions of the system of difference equations $x_{n+1}=\left(a+x_{n}\right) /\left(b+y_{n}\right), y_{n+1}=\left(d+y_{n}\right) /\left(e+x_{n}\right), n=0,1, \ldots$, where the parameters $a, b, d$, and $e$ are positive numbers and the initial conditions $x_{0}$ and $y_{0}$ are arbitrary nonnegative numbers. In certain range of parameters, we prove the existence of the global stable manifold of the unique positive equilibrium of this system which is the graph of an increasing curve. We show that the stable manifold of this system separates the positive quadrant of initial conditions into basins of attraction of two types of asymptotic behavior. In the case where $a=d$ and $b=e$, we find an explicit equation for the stable manifold to be $y=x$.

Copyright (c) 2006 M. R. S. Kulenović and M. Nurkanović. This is an open access article distributed under the Creative Commons Attribution License, which permits unrestricted use, distribution, and reproduction in any medium, provided the original work is properly cited.

\section{Introduction and preliminaries}

The following system of difference equations was considered in [12]:

$$
x_{n+1}=\frac{a+x_{n}}{b+y_{n}}, \quad y_{n+1}=\frac{d+y_{n}}{e+x_{n}}, \quad n=0,1, \ldots,
$$

where the parameters $a, b, d$, and $e$ are positive numbers and the initial conditions $x_{0}$ and $y_{0}$ are arbitrary nonnegative numbers.

It has been shown in [12] that (1.1) has the unique positive equilibrium which is globally asymptotically stable in the following three cases:

(1) $b>1, e>1$;

(2) $b=1, e>1, a<d$;

(3) $b>1, e=1, a>d$.

It has been also shown in [12] that (1.1) has the unique positive equilibrium $E=(\bar{x}, \bar{y})$ which is a saddle point in the following three cases: 
2 Competitive system of rational difference equations

(4) $b<1, e<1$;

(5) $b=1, e<1, a>d$;

(6) $b<1, e=1, d>a$.

We also proved that all solutions of (1.1) that start in certain regions $\left(x_{0}, y_{0}\right) \in O_{i} \backslash E$ $(i=1,2)$ approach $\{(\infty, 0)\}$ or $\{(0, \infty)\}$ as $n \rightarrow \infty$. Here $O_{i}$ denotes a region in the first quadrant and depends on the case (Lemma 2.2).

For each $v \in \mathbb{R}_{+}^{2}$, define $Q_{i}(v)$ for $i=1, \ldots, 4$ to be the usual four quadrants based at $v$ and numbered in a counterclockwise direction, for example, $Q_{1}(v)=\left\{(x, y) \in \mathbb{R}_{+}^{2}: v_{1} \leq\right.$ $\left.x, v_{2} \leq y\right\}$.

In cases (4)-(6) we believe that the global stable manifold $W^{s}(E) \subset Q_{1}(E) \cup Q_{3}(E)$ of $E$ separates the positive quadrant and serves as a threshold for mutual exclusion, that is, for all orbits below this manifold the $y$ sequence converges to zero and the $x$ sequence becomes unbounded and for all orbits above this manifold the $x$ sequence converges to zero and the $y$ sequence becomes unbounded.

Precisely, we have the following conjecture that was formulated in [12].

Conjecture 1.1. Each orbit in Int $\mathbb{R}_{+}^{2}$ starting above $W^{s}(E)$ remains above $W^{s}(E)$ and is asymptotic to $\{(0, \infty)\}$, that is, $\lim _{n \rightarrow \infty} x_{n}=0, \lim _{n \rightarrow \infty} y_{n}=\infty$. Each orbit in Int $\mathbb{R}_{+}^{2}$ starting below $W^{s}$ remains below $W^{s}$ and is asymptotic to $\{(\infty, 0)\}$, that is, $\lim _{n \rightarrow \infty} x_{n}=\infty$, $\lim _{n \rightarrow \infty} y_{n}=0$.

The goals of this paper are to prove this conjecture and to prove the result on the rate of convergence of solutions of (1.1) in the cases of global asymptotic stability (1)-(3). Thus we will show that in some cases where the unique positive equilibrium is a saddle point the principle of competitive exclusion applies. In fact we believe that in the case of a saddle point the local behavior implies the global behavior for competitive linear fractional systems.

As a biological model, system (1.1) may represent the competition between two populations which reproduce in discrete generations. The phase variables $x_{n}$ and $y_{n}$ denote population sizes during the $n$th generation and the sequence $\left\{\left(x_{n}, y_{n}\right): n=0,1,2, \ldots\right\}$ represents population changes from one generation to the next. Since the transition function for each population is a decreasing function of the other population's size, the populations are competing with one another.

Competition between 2-species with rational transition functions has been studied by Hassell and Comins [7], Franke and Yakubu [5, 6], Selgrade and Ziehe [16], Smith [17], and others. A simple competitive model that allows unbounded growth of a population size has been discussed in $[1,2]$ :

$$
x_{n+1}=\frac{x_{n}}{A+y_{n}}, \quad y_{n+1}=\frac{y_{n}}{B+x_{n}}, \quad n=0,1, \ldots
$$

See also $[11,17]$. In [2] we show that when $A<1, B<1$, the stable manifold of the positive equilibrium $(1-B, 1-A)$ of system (1.2) separates the positive quadrant into basins of attraction of two types of asymptotic behavior. From a biological perspective, $W^{s}((1-B, 1-A))$ is a threshold manifold which separates the regions of species extinction and so the competitive exclusion principle holds. For other values of parameters we 
have obtained different asymptotic results ranging from very simple behavior where all solutions are converging to $(0,0)$ when $A>1, B>1$, to the case of an infinite number of nonhyperbolic equilibrium points when $A=1$ or $B=1$. In the last case there are still some open problems about the global behavior of system (1.2). See [1].

In [3] we investigated the effect of help that only one population receives, that is, we consider

$$
x_{n+1}=\frac{x_{n}+h}{A+y_{n}}, \quad y_{n+1}=\frac{y_{n}}{B+x_{n}}, \quad n=0,1, \ldots
$$

In [12] we investigated the effect of the parameters $h_{1}, h_{2}>0$ which represent the sizes of immigration or help that populations $x$ and $y$ respectively receive. In this case we describe the dynamics with

$$
X_{n+1}=\frac{X_{n}}{A+Y_{n}}+h_{1}, \quad Y_{n+1}=\frac{Y_{n}}{B+X_{n}}+h_{2}, \quad n=0,1, \ldots
$$

where $h_{1}, h_{2}>0$. Using the substitutions $u_{n}=X_{n}-h_{1}, v_{n}=Y_{n}-h_{2}$, system (1.4) is reduced to

$$
u_{n+1}=\frac{h_{1}+u_{n}}{A+h_{2}+v_{n}}, \quad v_{n+1}=\frac{h_{2}+v_{n}}{B+h_{1}+u_{n}}, \quad n=0,1, \ldots,
$$

which is of the form (1.1). In [12] we showed that in cases (1)-(3) the introduction of the positive parameters $a$ and $d$ creates a unique positive equilibrium which is globally asymptotically stable, and so the principle of competitive coexistence applies. The corresponding system (1.2) in case (1) has the property that the zero equilibrium is globally asymptotically stable. In fact we believe that the local asymptotic stability implies the global asymptotic stability for competitive linear fractional systems. We will formulate this statement as a conjecture.

In [12] we showed that in cases (5) and (6) an introduction of the positive parameters $a$ and $d$ changed the global behavior of system (1.2) while in case (4) the global qualitative behavior of (1.2) does not seem to be affected by $a$ and $d$.

We now give some basic notions about systems and maps in the plane of the form:

$$
x_{n+1}=f\left(x_{n}, y_{n}\right), \quad y_{n+1}=g\left(x_{n}, y_{n}\right), \quad n=0,1,2, \ldots
$$

Consider a map $\mathbf{F}=(f, g)$ on a set $\mathscr{R} \subset \mathbb{R}^{2}$, and let $E \in \mathscr{R}$. The point $E \in \mathscr{R}$ is called a fixed point if $\mathbf{F}(E)=E$. An isolated fixed point is a fixed point that has a neighborhood with no other fixed points in it. A fixed point $E \in \mathscr{R}$ is an attractor if there exists a neighborhood $U$ of $E$ such that $\mathbf{F}^{n}(\mathbf{x}) \rightarrow E$ as $n \rightarrow \infty$ for $\mathbf{x} \in \mathcal{U}$; the basin of attraction is the set of all $\mathbf{x} \in \mathscr{R}$ such that $\mathbf{F}^{n}(\mathbf{x}) \rightarrow E$ as $n \rightarrow \infty$. A fixed point $E$ is a global attractor on a set $\mathscr{K}$ if $E$ is an attractor and $\mathscr{Y}$ is a subset of the basin of attraction of $E$. If $\mathbf{F}$ is differentiable at a fixed point $E$, and if the Jacobian $J_{\mathrm{F}}(E)$ has one eigenvalue with modulus less than one and a second eigenvalue with modulus greater than one, $E$ is said to be a saddle. See [15] for additional definitions. 
4 Competitive system of rational difference equations

Definition 1.2. Let $\mathbf{F}=(f, g)$ be a continuously differentiable function and let $U$ be a neighborhood of a saddle point $(\bar{x}, \bar{y})$ of $(1.6)$. The local stable manifold $W_{\text {loc }}^{s}$ is the set

$$
W_{\mathrm{loc}}^{s}((\bar{x}, \bar{y}))=\left\{(x, y): \mathbf{F}^{n}(x, y) \in U \forall n \geq 0, \lim _{n \rightarrow \infty} \mathbf{F}^{n}(x, y)=(\bar{x}, \bar{y})\right\}
$$

The global stable manifold $W^{s}$ of a saddle point $(\bar{x}, \bar{y})$ is the set

$$
W^{s}((\bar{x}, \bar{y}))=\left\{(x, y): \lim _{n \rightarrow \infty} \mathbf{F}^{n}(x, y)=(\bar{x}, \bar{y})\right\}
$$

The main result in the linearized stability analysis is the following result $[10,15]$.

Theorem 1.3 (linearized stability theorem). Let $\mathbf{F}=(f, g)$ be a continuously differentiable function defined on an open set $W$ in $\mathbb{R}^{2}$, and let $E=(\bar{x}, \bar{y})$ in $W$ be a fixed point of $\mathbf{F}$.

(a) If all the eigenvalues of the Jacobian matrix $J_{\mathrm{F}}(E)$ have modulus less than one, then the equilibrium point $E$ of (1.6) is asymptotically stable.

(b) If at least one of the eigenvalues of the Jacobian matrix $J_{\mathrm{F}}(E)$ has modulus greater than one, then the equilibrium point $E$ of (1.6) is unstable.

(c) All the eigenvalues of the Jacobian matrix $J_{\mathrm{F}}(E)$ have modulus less than one if and only if every solution of the characteristic equation

$$
\lambda^{2}-\operatorname{Tr} J_{\mathbf{F}}(E) \lambda+\operatorname{Det} J_{\mathbf{F}}(E)=0
$$

lies inside unit circle, that is, if and only if

$$
\left|\operatorname{Tr} J_{\mathrm{F}}(E)\right|<1+\operatorname{Det} J_{\mathrm{F}}(E)<2
$$

Here we give some basic facts about the monotone maps in the plane, see $[2,3,8,17]$. Now, we write system (1.1) in the form

$$
\left(\begin{array}{l}
x \\
y
\end{array}\right)_{n+1}=T\left(\begin{array}{l}
x \\
y
\end{array}\right)_{n},
$$

where the map $T$ is given as

$$
T:\left(\begin{array}{l}
x \\
y
\end{array}\right) \longrightarrow\left(\begin{array}{l}
\frac{a+x}{b+y} \\
\frac{d+y}{e+x}
\end{array}\right)=\left(\begin{array}{l}
f(x, y) \\
g(x, y)
\end{array}\right)
$$

The map $T$ may be viewed as a monotone map if we define a partial order on $\mathbb{R}^{2}$ so that the positive cone in this new partial order is the fourth quadrant. Specifically, for $v=\left(v_{1}, v_{2}\right), w=\left(w_{1}, w_{2}\right) \in \mathbb{R}^{2}$, we say that $v \leq w$ if $v_{1} \leq w_{1}$ and $w_{2} \leq v_{2}$. Two points $v, w \in \mathbb{R}_{+}^{2}$ are said to be related if $v \leq w$ or $w \leq v$. Also, a strict inequality between points may be defined as $v<w$ if $v \leq w$ and $v \neq w$. A stronger inequality may be defined as $v \ll w$ if $v_{1}<w_{1}$ and $w_{2}<v_{2}$. A map $f: \operatorname{Int} \mathbb{R}_{+}^{2} \rightarrow \operatorname{Int} \mathbb{R}_{+}^{2}$ is strongly monotone if $v<w$ implies that $f(v) \ll f(w)$ for all $v, w \in \operatorname{Int} \mathbb{R}_{+}^{2}$. Clearly, being related is an invariant under iteration of 
a strongly monotone map. Differentiable strongly monotone maps have Jacobian with constant sign configuration

$$
\left[\begin{array}{ll}
+ & - \\
- & +
\end{array}\right] \text {. }
$$

The mean value theorem and the convexity of $\mathbb{R}_{+}^{2}$ may be used to show that $T$ is monotone, as in [2].

The following result gives the rate of convergence of solutions of a system of difference equations

$$
\mathbf{x}_{n+1}=[A+B(n)] \mathbf{x}_{n},
$$

where $\mathbf{x}_{n}$ is a $k$-dimensional vector, $A \in \mathbf{C}^{k \times k}$ is a constant matrix, and $B: \mathbf{Z}^{+} \rightarrow \mathbf{C}^{k \times k}$ is a matrix function satisfying

$$
\|B(n)\| \longrightarrow 0 \quad \text { when } n \longrightarrow \infty
$$

where $\|\cdot\|$ denotes any matrix norm which is associated with the vector norm; $\|\cdot\|$ denotes the Euclidean norm in $\mathbb{R}^{2}$ given by

$$
\|(x, y)\|=\sqrt{x^{2}+y^{2}} .
$$

Theorem 1.4. Assume that condition (1.15) holds. If $\mathbf{x}$ is a solution of (1.14), then

$$
\lim _{n \rightarrow \infty} \sqrt[n]{\| \mathbf{x}_{n}||}=\left|\lambda_{i}(A)\right|, \quad i=1, \ldots, k
$$

where $\lambda_{i}(A)$ denotes one of the eigenvalues of the matrix $A$.

\section{Proof of conjecture}

Proof of Conjecture 1.1 will be given mainly in case (4) (proofs in the remaining cases (5) and (6) are analogous).

Define the sets $S_{1}$ and $S_{2}$ as follows:

$$
\begin{aligned}
& S_{1}=\left\{(x, y) \in \mathbb{R}_{+}^{2}: \frac{d}{x+e-1} \leq y \leq \frac{a}{x}+1-b\right\} ; \\
& S_{2}=\left\{(x, y) \in \mathbb{R}_{+}^{2}: \frac{a}{y+b-1} \leq x \leq \frac{d}{y}+1-e\right\} .
\end{aligned}
$$

Set

$$
\begin{aligned}
\phi_{1}(x) & =\frac{d}{x+e-1}, & \phi_{2}(x) & =\frac{a}{x}+1-b, \\
\psi_{1}(y) & =\frac{a}{y+b-1}, & \psi_{2}(y) & =\frac{d}{y}+1-e .
\end{aligned}
$$


Note that for $x>\bar{x}, y>\bar{y}: \phi_{i}(x) \in Q_{4}(E), \psi_{i}(y) \in Q_{2}(E)(i=1,2)$, and that for $(x, y) \in S_{1}$, $x>\bar{x}: \phi_{1}(x)<y<\phi_{2}(x)<\bar{y}$, while for $(x, y) \in S_{2}, y>\bar{y}: \psi_{1}(y)<x<\psi_{2}(y)<\bar{x}$. Consequently, $S_{1} \subset Q_{4}(E)$ and $S_{2} \subset Q_{2}(E)$.

The following two results were proved in [12].

LEMMA 2.1. $S_{1}$ and $S_{2}$ are invariant sets.

Lemma 2.2. Assume that $b<1$ and $e<1$.

(1) Set $S_{1}$, defined by (2.1), is an invariant set of (1.1) and every solution $\left\{\left(x_{n}, y_{n}\right)\right\}$ of (1.1) with initial conditions $\left(x_{0}, y_{0}\right) \in S_{1} \backslash$ E satisfies

$$
\lim _{n \rightarrow \infty} x_{n}=\infty, \quad \lim _{n \rightarrow \infty} y_{n}=0, \quad \mathscr{B}((\infty, 0)) \supseteq S_{1} \backslash E .
$$

(2) Set $S_{2}$, defined by (2.2), is an invariant set of (1.1) and every solution $\left\{\left(x_{n}, y_{n}\right)\right\}$ of (1.1) with initial conditions $\left(x_{0}, y_{0}\right) \in S_{2} \backslash$ E satisfies

$$
\lim _{n \rightarrow \infty} x_{n}=0, \quad \lim _{n \rightarrow \infty} y_{n}=\infty, \quad \mathscr{B}((0, \infty)) \supseteq S_{2} \backslash E .
$$

Here $\mathscr{B}(S)$ denotes the basin of attraction of a set $S$, see $[4,10,15]$.

Next, we will prove the following result.

Lemma 2.3. If $\left(x_{0}, y_{0}\right) \in Q_{4}(E) \backslash E$, then $\left(x_{n}, y_{n}\right) \in \operatorname{Int} Q_{4}(E)$ for all $n \geq 1$ and $\left(x_{n}, y_{n}\right) \rightarrow$ $\{(\infty, 0)\}$ as $n \rightarrow \infty$.

Proof. If $\left(x_{0}, y_{0}\right) \in Q_{4}(E) \backslash E$, then $E<\left(x_{0}, y_{0}\right)$ and so

$$
E=T(E) \ll T\left(\left(x_{0}, y_{0}\right)\right)=\left(x_{1}, y_{1}\right) \Longrightarrow\left(x_{1}, y_{1}\right) \in \operatorname{Int} Q_{4}(E) .
$$

By induction

$$
\left(x_{n}, y_{n}\right) \in \operatorname{Int} Q_{4}(E) \quad \forall n \geq 1 .
$$

Since $\left(x_{1}, y_{1}\right) \in \operatorname{Int} Q_{4}(E)$, there is $u \in S_{1}$ so that $u<\left(x_{1}, y_{1}\right)$. Lemma 2.2 implies $T^{n}(u) \rightarrow$ $\{(\infty, 0)\}$ as $n \rightarrow \infty$. Since $T^{n}(u)<T^{n}\left(\left(x_{1}, y_{1}\right)\right)$ for all $n \geq 1$, it follows that $\left(x_{n}, y_{n}\right) \rightarrow$ $\{(\infty, 0)\}$ as $n \rightarrow \infty$.

Lemma 2.4. If $\left(x_{0}, y_{0}\right) \in Q_{2}(E) \backslash E$, then $\left(x_{n}, y_{n}\right) \in \operatorname{Int} Q_{2}(E)$ for all $n \geq 1$ and $\left(x_{n}, y_{n}\right) \rightarrow$ $\{(0, \infty)\}$ as $n \rightarrow \infty$.

Proof. If $\left(x_{0}, y_{0}\right) \in Q_{2}(E) \backslash E$, then $\left(x_{0}, y_{0}\right)<E$ and so

$$
T\left(\left(x_{0}, y_{0}\right)\right)=\left(x_{1}, y_{1}\right) \ll E=T(E) \Longrightarrow\left(x_{1}, y_{1}\right) \in \operatorname{Int} Q_{4}(E) .
$$

By induction

$$
\left(x_{n}, y_{n}\right) \in \operatorname{Int} Q_{2}(E) \quad \forall n \geq 1 .
$$

Since $\left(x_{1}, y_{1}\right) \in \operatorname{Int} Q_{2}(E)$, there is $v \in S_{1}$ so that $\left(x_{1}, y_{1}\right)<v$. Lemma 2.2 asserts $T^{n}(v) \rightarrow$ $\{(0, \infty)\}$ as $n \rightarrow \infty$. Since $T^{n}\left(\left(x_{1}, y_{1}\right)\right)<T^{n}(v)$ for all $n \geq 1$, it follows that $\left(x_{n}, y_{n}\right) \rightarrow$ $\{(0, \infty)\}$ as $n \rightarrow \infty$. 
Thus we see that sets $Q_{2}(E)$ and $Q_{4}(E)$ are invariant sets of system (1.1).

Proposition 2.5. The global stable manifold $W^{s}$ of $E$ is subset of $\operatorname{Int} Q_{1}(E) \cup \operatorname{Int} Q_{3}(E) \cup E$ and $W^{s}$ contains no related points.

Proof. If two points in $W^{s}$ are related, then all their iterations are related as well because of monotonicity of $T$. In particular, the iterations in $W_{\text {loc }}^{s}$ would be related. Therefore it is enough to establish the result for $W_{\text {loc }}^{s}$. In view of Lemmas 2.3 and 2.4, we have that $W^{s} \subset \operatorname{Int} Q_{1}(E) \cup \operatorname{Int} Q_{3}(E) \cup E$. Moreover, the stable eigenvector in $E$ can be chosen to have positive component which implies that there exists strictly increasing function $H(x)$ such that $W_{\text {loc }}^{s}$ is a graph of $H$. The rest of the proof is identical to the proof of [2, Proposition 3.2].

Theorem 2.6. System (1.1) has no prime period-two solution.

Proof. Set

$$
T(x, y)=\left(\frac{a+x}{b+y}, \frac{d+y}{e+x}\right)
$$

Then

$$
\begin{aligned}
T(T(x, y)) & =T\left(\left(\frac{a+x}{b+y}, \frac{d+y}{e+x}\right)\right)=\left(\frac{a+(a+x) /(b+y)}{b+(d+y) /(e+x)}, \frac{d+(d+y) /(e+x)}{e+(a+x) /(b+y)}\right) \\
& =\left(\frac{(a b+a y+a+x)(e+x)}{(b e+b x+d+y)(b+y)}, \frac{(d e+d x+d+y)(b+y)}{(e b+e y+a+x)(e+x)}\right) .
\end{aligned}
$$

Period-two solutions satisfy

$$
\begin{aligned}
& \frac{(a b+a y+a+x)(e+x)}{(b e+b x+d+y)(b+y)}-x=0, \\
& \frac{(d e+d x+d+y)(b+y)}{(e b+e y+a+x)(e+x)}-y=0 .
\end{aligned}
$$

Solution of this system is equilibrium point and

$$
x=-\frac{(e-1)\left(\rho\left(b e^{2}-e^{2}+a e-a b-b+1\right)+d(e+1)(1-b)\right)}{d(e-b)(1-b)+\rho\left(b e^{2}-e^{2}-a b+b d+1+a e-d e-b\right)}, \quad y=\rho,
$$

where $\rho$ is a root of

$$
A Z^{2}+B Z+C=0,
$$

and where

$$
\begin{gathered}
A=(1+e)(b e-1), \\
B=(1+b)(1+e)(-e+b e+b-1+a-d), \\
C=(1+b)\left(b^{2}+b^{2} e-b d+a b-b d e-1+a-e\right) .
\end{gathered}
$$


We will show that either one of the roots of (2.14) is negative, or both are complex conjugate. We will give the proof in all three cases (4)-(6).

Case $1(b<1, e<1)$. In this case $A<0$ and $B$ and $C$ could be of arbitrary sign. We will consider all three possibilities for $C$.

$\left(1^{\circ}\right)$

$$
\begin{aligned}
C>0 & \Longleftrightarrow b^{2}+b^{2} e-b d+a b-b d e-1+a-e>0 \\
& \Longleftrightarrow a(b+1)>(1+e)\left(b d+1-b^{2}\right) \Longleftrightarrow a>\frac{(1+e)\left(b d+1-b^{2}\right)}{b+1} .
\end{aligned}
$$

In this case we have

$$
Z_{1} Z_{2}=\frac{C}{A}<0
$$

which shows that one of the roots of $(2.14)$ is negative.

$\left(2^{\circ}\right)$

$$
C=0 \Longleftrightarrow a=\frac{(1+e)\left(b d+1-b^{2}\right)}{b+1} .
$$

In this case (2.14) takes the form $A Z^{2}+B Z=0$, which implies $Z_{1}=0$ and $Z_{2}=-B / A$. Now we have

$$
\begin{aligned}
B & =(1+b)(1+e)\left(-e+b e+b-1+\frac{(1+e)\left(b d+1-b^{2}\right)}{b+1}-d\right) \\
& =(1+e)(b e-1) d
\end{aligned}
$$

which implies that $Z_{2}=-B / A=-d<0$, which completes the proof in this case.

$\left(3^{\circ}\right)$

$$
C<0 \Longleftrightarrow a<\frac{(1+e)\left(b d+1-b^{2}\right)}{b+1} .
$$

In this case we have $Z_{1} Z_{2}=C / A>0$, which implies that $Z_{1}$ and $Z_{2}$ are either real and of same sign or are complex conjugate. As in case $\left(2^{\circ}\right)$, we obtain

$$
B<(1+e)(b e-1) d<0 .
$$

Thus, either $Z_{1}$ and $Z_{2}$ are complex conjugate or negative which proves lemma in this case.

Case $2(b=1, e<1, a>d)$. Now we have

$$
\begin{gathered}
A=(e+1)(e-1)=e^{2}-1<0, \\
B=2(1+e)(a-d)>0, \\
C=2(a-d+a-d e)>2(a-d+a-d)=4(a-d)>0 .
\end{gathered}
$$

The corresponding discriminant has the form

$$
D=B^{2}-4 A C=4(e+1)\left((e+1)(a-d)^{2}+8(1-e)(a-d+a-d e)\right)>0,
$$


which shows that the roots are real and different. In view of Viet's formulas, we have

$$
Z_{1} Z_{2}=\frac{C}{A}=\frac{2(2 a-d-d e)}{e^{2}-1}<0 \Longrightarrow \operatorname{sign} Z_{1}=-\operatorname{sign} Z_{2}
$$

which completes the proof in this case.

Case $3(b<1, e=1, d>a)$. In this case we have

$$
\begin{gathered}
A=2(b-1), \\
B=2(b+1)(2 b-2+a-d), \\
C=(b+1)\left(2 b^{2}-2 b d+a b+a-2\right) .
\end{gathered}
$$

Clearly, $A<0$ and $B<0$ which implies

$$
Z_{1}+Z_{2}=-\frac{B}{A}<0
$$

If the solutions of (2.14) are complex conjugate, the proof of lemma is completed. If the solutions of (2.14) are real, then the last inequality implies that at least one of the term of period-two solution is negative which is impossible.

The proof of next result is similar to the proof of [2, Proposition 3.3] and it will be omitted. This proof makes essential use of the nonexistence of prime period-two solution that was proved in Theorem 2.6.

Proposition 2.7. Assume that $b<1$ and $e<1$. The global stable manifold $W^{s}$ of E separates the positive quadrant $\mathbb{R}_{+}^{2}$, that is, the portion of $W^{s}$ in $Q_{3}(E)$ connects $E$ with some point on the $x$-axis or on the $y$-axis and the portion of $W^{s}$ in $Q_{1}(E)$ is unbounded.

We now state the major result of this section. The proof of this result is similar to the proof of [2, Theorem 3.1] and will be omitted.

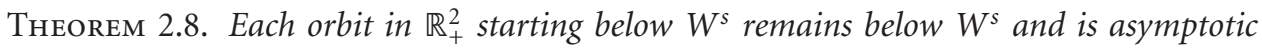
to $\{(\infty, 0)\}$. Each orbit in $\mathbb{R}_{+}^{2}$ starting above $W^{s}$ remains above $W^{s}$ and is asymptotic to $\{(0, \infty)\}$.

In the special case $a=d, b=e$, we will show that the global stable manifold $W^{s}(E)$ is the bisector $y=x$.

Theorem 2.9. Let $a, b \in(0,1)$ and $a=d, b=e$. The line $y=x$ is the global stable manifold $W^{s}(E)$. Each orbit starting above $W^{s}$ remains above $W^{s}$ and is asymptotic to $\{(0, \infty)\}$, and each orbit starting below $W^{s}$ remains below $W^{s}$ and is asymptotic to $\{(\infty, 0)\}$.

Proof. When $a=d, b=e,(1.1)$ becomes

$$
x_{n+1}=\frac{a+x_{n}}{b+y_{n}}, \quad y_{n+1}=\frac{a+y_{n}}{b+x_{n}}, \quad n=0,1, \ldots
$$


To prove the first statement we need to show that the line $y=x$ is an invariant set, that is, $T_{a}(\{(x, x): x \geq 0\}) \subseteq\{(x, x): x \geq 0\}$, where

$$
T_{a}(x, y)=\left(\frac{a+x}{b+y}, \frac{a+y}{b+x}\right)
$$

and that $\left\{\left(x_{n}, y_{n}\right)\right\} \rightarrow E$ as $n \rightarrow \infty$ for every solution $\left\{\left(x_{n}, y_{n}\right)\right\}$ of (2.27) initiated on the line $y=x$. Taking $x_{0}=y_{0}$ it is obvious that $x_{1}=y_{1}$, and induction yields $x_{n}=y_{n}$, $n=0,1, \ldots$ In this case the system (2.27) reduces to a single Riccati difference equation

$$
x_{n+1}=\frac{a+x_{n}}{b+x_{n}} .
$$

The Riccati number, see [9], for this equation is

$$
R=\frac{b-a}{(b+1)^{2}}<\frac{1}{4}
$$

and so every solution of (2.29) tends to the equilibrium $E$ (see [9]). The closed-form solution to this equation can be obtained (see [9]). Using the uniqueness of the stable manifold (see [15, page 182]) and the fact that the asymptotic behavior off the line $x=y$ follows from Theorem 2.8, it follows that $y=x$ is the global stable manifold.

Remark 2.10. The results of this paper show that in the cases (4)-(6) the competitive exclusion principle applies and so one of the species goes extinct. The results of [12] showed that in the cases (1)-(3) the competitive coexistence principle applies.

In fact, based on our results in this paper and the results of [12], we formulate the following conjecture.

Conjecture 2.11. (1) The statement of Conjecture 1.1 holds whenever the unique interior equilibrium point $E$ of (1.1) is a saddle point.

(2) The unique interior equilibrium point $E$ of (1.1) is a global attractor and so globally asymptotically stable whenever $E$ is locally asymptotically stable.

In other words, Conjecture 2.11 states that the global dynamics of (1.1) is determined by its local dynamics. It would be interesting to find the most general class of competitive systems (1.6) for which the global dynamics is determined by its local dynamics. This would provide a partial answer to May's problem [13]. The significance of our results is that we are establishing an important step toward the solution of this problem.

\section{Rate of convergence}

In this section we will determine the rate of convergence of a solution that converges to the equilibrium $E$ in cases (1)-(3).

Assume that a solution $\left\{\left(x_{n}, y_{n}\right)\right\}$ converges to $E$. Then $\lim _{n \rightarrow \infty} x_{n}=\bar{x}$ and $\lim _{n \rightarrow \infty} y_{n}=$ $\bar{y}$. 
First we will find a system of limiting equations for the map T. The error terms are given as

$$
\begin{aligned}
& x_{n+1}-\bar{x}=\frac{a+x_{n}}{b+y_{n}}-\frac{a+\bar{x}}{b+\bar{y}}=\frac{1}{b+y_{n}}\left(x_{n}-\bar{x}\right)-\frac{a+\bar{x}}{b+\bar{y}} \cdot \frac{1}{b+y_{n}}\left(y_{n}-\bar{y}\right), \\
& y_{n+1}-\bar{y}=\frac{d+y_{n}}{e+x_{n}}-\frac{d+\bar{y}}{e+\bar{x}}=-\frac{1}{e+x_{n}} \cdot \frac{d+\bar{y}}{e+\bar{x}}\left(x_{n}-\bar{x}\right)+\frac{1}{e+x_{n}}\left(y_{n}-\bar{y}\right),
\end{aligned}
$$

that is,

$$
\begin{aligned}
& x_{n+1}-\bar{x}=\frac{1}{b+y_{n}}\left(x_{n}-\bar{x}\right)-\frac{\bar{x}}{b+y_{n}}\left(y_{n}-\bar{y}\right), \\
& y_{n+1}-\bar{y}=-\frac{\bar{y}}{e+x_{n}}\left(x_{n}-\bar{x}\right)+\frac{1}{e+x_{n}}\left(y_{n}-\bar{y}\right) .
\end{aligned}
$$

Set $e_{n}^{1}=x_{n}-\bar{x}$ and $e_{n}^{2}=y_{n}-\bar{y}$. System (3.2) can be represented as

$$
e_{n+1}^{1}=a_{n} e_{n}^{1}+b_{n} e_{n}^{2}, \quad e_{n+1}^{2}=c_{n} e_{n}^{1}+d_{n} e_{n}^{2},
$$

where

$$
a_{n}=\frac{1}{b+y_{n}}, \quad b_{n}=-\frac{\bar{x}}{b+y_{n}}, \quad c_{n}=-\frac{\bar{y}}{e+x_{n}}, \quad d_{n}=\frac{1}{e+x_{n}} .
$$

Taking the limits of $a_{n}, b_{n}, c_{n}$, and $d_{n}$, we obtain (case $b>1, e>1$ )

$$
\lim _{n \rightarrow \infty} a_{n}=\frac{1}{b+\bar{y}}, \quad \lim _{n \rightarrow \infty} b_{n}=-\frac{\bar{x}}{b+\bar{y}}, \quad \lim _{n \rightarrow \infty} c_{n}=-\frac{\bar{y}}{e+\bar{x}}, \quad \lim _{n \rightarrow \infty} d_{n}=\frac{1}{e+\bar{x}} .
$$

In case (2), when $b=1, e>1, a<d$, and $E=(a(e-1) /(d-a),(d-a) /(e-1))$, we have

$$
\begin{gathered}
\lim _{n \rightarrow \infty} a_{n}=\frac{e-1}{e-1+d-a}, \quad \lim _{n \rightarrow \infty} b_{n}=-\frac{a(e-1)^{2}}{(d-a)(e-1+d-a)}, \\
\lim _{n \rightarrow \infty} c_{n}=-\frac{(d-a)^{2}}{(e-1)(e d-a)}, \quad \lim _{n \rightarrow \infty} d_{n}=\frac{d-a}{e d-a} .
\end{gathered}
$$

Finally, in the case (3), when $b>1, e=1, a>d$, and $E=((a-d) /(b-1), d(b-1) /$ $(a-d))$, we obtain

$$
\begin{gathered}
\lim _{n \rightarrow \infty} a_{n}=\frac{a-d}{a b-d}, \quad \lim _{n \rightarrow \infty} b_{n}=-\frac{(a-d)^{2}}{(b-1)(a b-d)}, \\
\lim _{n \rightarrow \infty} c_{n}=-\frac{d(b-1)^{2}}{(a-d)(a+b-1-d)}, \quad \lim _{n \rightarrow \infty} d_{n}=\frac{b-1}{a+b-1-d} .
\end{gathered}
$$


Now the limiting system of error terms can be written as

$$
\left(\begin{array}{c}
e_{n+1}^{1} \\
e_{n+1}^{2}
\end{array}\right)=\left(\begin{array}{cc}
\frac{1}{b+\bar{y}} & -\frac{\bar{x}}{b+y_{n}} \\
-\frac{\bar{y}}{e+x_{n}} & \frac{1}{e+x_{n}}
\end{array}\right)\left(\begin{array}{l}
e_{n}^{1} \\
e_{n}^{2}
\end{array}\right)
$$

in the case (1), and as

$$
\left(\begin{array}{l}
e_{n+1}^{1} \\
e_{n+1}^{2}
\end{array}\right)=\left(\begin{array}{cc}
\frac{e-1}{e-1+d-a} & -\frac{a(e-1)^{2}}{(d-a)(e-1+d-a)} \\
-\frac{(d-a)^{2}}{(e-1)(e d-a)} & \frac{d-a}{e d-a}
\end{array}\right)\left(\begin{array}{l}
e_{n}^{1} \\
e_{n}^{2}
\end{array}\right),
$$

in the case (2). Finally, in the case (3)

$$
\left(\begin{array}{c}
e_{n+1}^{1} \\
e_{n+1}^{2}
\end{array}\right)=\left(\begin{array}{cc}
\frac{a-d}{a b-d} & -\frac{(a-d)^{2}}{(b-1)(a b-d)} \\
-\frac{d(b-1)^{2}}{(a-d)(a+b-1-d)} & \frac{b-1}{a+b-1-d}
\end{array}\right)\left(\begin{array}{l}
e_{n}^{1} \\
e_{n}^{2}
\end{array}\right) .
$$

This shows that all the systems are exactly the linearized systems of (1.1) evaluated in the equilibrium $E$.

Using Theorem 1.4 and Pituk's result [14], we have the following result.

Theorem 3.1. Assume that a solution $\left\{\left(x_{n}, y_{n}\right)\right\}$ of (1.1) converges to $E$ (for instance, this is, true for all solutions when $b>1, e>1$, or $b=1, e>1, a<d$, or $b>1, e=1, a>d$ ). The error vector $\mathbf{e}_{n}=\left(\begin{array}{c}e_{n}^{1} \\ e_{n}^{2}\end{array}\right)$ of every solution of (1.1) satisfies both of the following asymptotic relations:

$$
\begin{aligned}
& \lim _{n \rightarrow \infty} \sqrt[n]{\left\|\mathbf{e}_{n}\right\|}=\left|\lambda_{1,2} J_{T}(E)\right|, \\
& \lim _{n \rightarrow \infty} \frac{\left\|\mathbf{e}_{n+1}\right\|}{\left\|\mathbf{e}_{n}\right\|}=\left|\lambda_{1,2} J_{T}(E)\right|,
\end{aligned}
$$

where $\lambda_{1,2} J_{T}(E)$ are the characteristic roots of matrix $J_{T}(E)$.

\section{References}

[1] D. Clark and M. R. S. Kulenović, A coupled system of rational difference equations, Computers \& Mathematics with Applications 43 (2002), no. 6-7, 849-867.

[2] D. Clark, M. R. S. Kulenović, and J. F. Selgrade, Global asymptotic behavior of a two-dimensional difference equation modelling competition, Nonlinear Analysis. Theory, Methods \& Applications 52 (2003), no. 7, 1765-1776.

[3] C. A. Clark, M. R. S. Kulenović, and J. F. Selgrade, On a system of rational difference equations, Journal of Difference Equations and Applications 11 (2005), no. 7, 565-580.

[4] S. N. Elaydi, Discrete Chaos, Chapman \& Hall/CRC, Florida, 2000.

[5] J. E. Franke and A.-A. Yakubu, Mutual exclusion versus coexistence for discrete competitive systems, Journal of Mathematical Biology 30 (1991), no. 2, 161-168. 
[6] Geometry of exclusion principles in discrete systems, Journal of Mathematical Analysis and Applications 168 (1992), no. 2, 385-400.

[7] M. P. Hassell and H. N. Comins, Discrete time models for two-species competition, Theoretical Population Biology 9 (1976), no. 2, 202-221.

[8] P. Hess and A. C. Lazer, On an abstract competition model and applications, Nonlinear Analysis. Theory, Methods \& Applications 16 (1991), no. 11, 917-940.

[9] M. R. S. Kulenović and G. Ladas, Dynamics of Second Order Rational Difference Equations with Open Problems and Conjectures, Chapman \& Hall/CRC, Florida, 2001.

[10] M. R. S. Kulenović and O. Merino, Discrete Dynamical Systems and Difference Equations with Mathematica, Chapman \& Hall/CRC, Florida, 2002.

[11] M. R. S. Kulenović and M. Nurkanović, Asymptotic behavior of a two dimensional linear fractional system of difference equations, Radovi Matematički 11 (2002), no. 1, 59-78.

[12] _ Asymptotic behavior of a system of linear fractional difference equations, Journal of Inequalities and Applications 2005 (2005), no. 2, 127-143.

[13] R. M. May, Stability in multispecies community models, Mathematical Biosciences 12 (1971), no. 1-2, 59-79.

[14] M. Pituk, More on Poincarés and Perron's theorems for difference equations, Journal of Difference Equations and Applications 8 (2002), no. 3, 201-216.

[15] C. Robinson, Dynamical Systems. Stability, Symbolic Dynamics, and Chaos, Studies in Advanced Mathematics, CRC Press, Florida, 1995.

[16] J. F. Selgrade and M. Ziehe, Convergence to equilibrium in a genetic model with differential viability between the sexes, Journal of Mathematical Biology 25 (1987), no. 5, 477-490.

[17] H. L. Smith, Planar competitive and cooperative difference equations, Journal of Difference Equations and Applications 3 (1998), no. 5-6, 335-357.

M. R. S. Kulenović: Department of Mathematics, University of Rhode Island, Kingston, RI 02881-0816, USA

E-mail address: kulenm@math.uri.edu

M. Nurkanović: Department of Mathematics, University of Tuzla, Tuzla 75000,

Bosnia and Herzegovina

E-mail address: mehmed.nurkanovic@untz.ba 